\title{
Lung ultrasound in the monitoring of COVID-19 infection
}

\author{
Author: Yale Tung-Chen ${ }^{\mathrm{A}}$
}

\section{Introduction}

Coronavirus disease 2019 (COVID-19) is a highly contagious illness caused by severe acute respiratory syndrome coronavirus 2 (SARS-CoV-2). Pneumonia and acute respiratory distress syndrome (ARDS) are the most common severe complications. There is growing evidence regarding the imaging findings of COVID-19 in chest X-rays and computed tomography (CT); however, their availability to clinical staff in this pandemic outbreak might be compromised. At this moment, the role of lung ultrasound (LUS) has yet to be explored. The purpose of this case report is to describe the natural course of the disease in mild infection managed at home.

Case report

We report a 35-year-old man with recently diagnosed COVID-19 infection. Clinical examination was unremarkable. The diagnosis of mild disease was made clinically which was later reaffirmed by LUS after identifying a bilateral small pleural effusion and a thickened pleural line. During follow up, subpleural consolidations appeared before symptoms slightly aggravated (cough, tiredness and fever). The patient's condition improved after adjustment of therapy at home.

Conclusion

LUS is an excellent tool in the characterisation of COVID-19

infection and is more available than $\mathrm{CT}$ or X-ray. We emphasise the utility and the opportunity that LUS presents in some clinical scenarios, like this COVID-19 pandemic, and how it may serve as a monitoring and therapy guide.

KEYWORDS: COVID-19, lung ultrasound, SARS-CoV-2

DOI: $10.7861 /$ clinmed.2020-0123

\section{Introduction}

A novel coronavirus, named severe acute respiratory syndrome coronavirus 2 (SARS-CoV-2), which causes the disease called coronavirus disease 2019 (COVID-19) has been identified as the cause of a series of pneumonia cases in Wuhan (China)

Author: ${ }^{\text {Ad }}$ irector of the emergency ultrasound division unit, Hospital Universitario La Paz, Madrid, Spain and associate professor, Universidad Alfonso X El Sabio, Madrid, Spain in December 2019.' On 11 March 2020, the World Health Organization declared the outbreak a pandemic, ${ }^{2}$ with the spread of the disease to more than 180 countries and almost all five continents, and dramatic increases in laboratory-confirmed cases.

The disease severity spectrum is believed to be broad, with many patients $(80 \%)$ being asymptomatic or mildly symptomatic, and mortality estimated to be less than $1 \%{ }^{3,4}$ Symptoms include fever, cough and malaise. ${ }^{4}$ Treatment is supportive as this condition is usually benign and has a limited course, and self-isolation at home is acceptable in these cases. However, pneumonia and acute respiratory distress syndrome (ARDS) are the most common severe complications. ${ }^{4}$

The purpose of this case report is to describe the sequential ultrasound findings and the associated clinical features of a mildly symptomatic COVID-19 patient in the home setting.

\section{Case report}

A previously healthy 35 -year-old man presented to the emergency department with a chief complaint of abrupt chills and malaise. He is an emergency physician and works treating COVID-19 patients in a tertiary hospital.

Due to his mild symptoms, after extracting the nasopharyngeal swab for a SARS-CoV-2 test, he was advised to stay isolated at home while waiting for the results, which came out 20 hours later as positive. At that time, a dry cough and oppressive bilateral headache started. His temperature at that moment was $37.4^{\circ} \mathrm{C}$. Physical exam was unremarkable, with normal lung auscultation. A bedside lung ultrasound (LUS) was performed with a hand-held ultrasound device (Butterfly $\mathrm{iQ}$ ), following an eight-zone scanning scheme $^{5}$ of the antero-lateral chest and then the posterior chest, and showed a normal A-line pattern of the lungs (Fig 1). The LUS was repeated daily and demonstrated the presence of small pleural effusion on both posterior lower lobes on day 2 with a thickened and irregular pleural line (Fig 2) when symptoms had started to improve. By day 4, when symptoms began to worsen, LUS showed left subpleural consolidations (Fig 3) with resolution of right-side abnormalities. At the same time, his cough became worse, low-grade fever reappeared $\left(37.2^{\circ} \mathrm{C}\right)$ and malaise increased significantly. Through the entire course of the disease, the lung sliding was present. During the following days, B-lines spread bilaterally in lateral and posterior zones (Fig 4). This persisted for a week and improved in the second week, while an A-line pattern was present in the anterior zones. The small pleural effusion resolved by day 13 .

Since the patient did not report any shortness of breath or chest pain, and had strictly mild symptoms, he kept a symptom and 


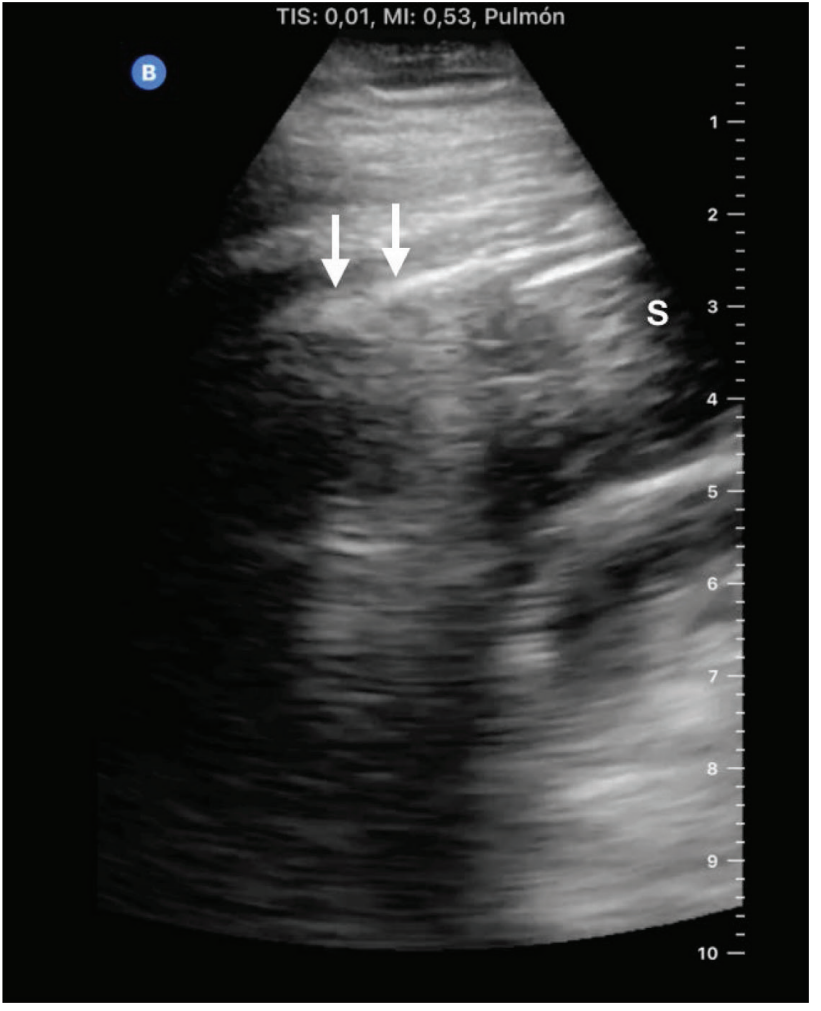

Fig 1. Day 1 - curvilinear probe. Left posterior lower lobe posterior basal segment of the lung. Normal lung sliding (arrows). S: spleen.

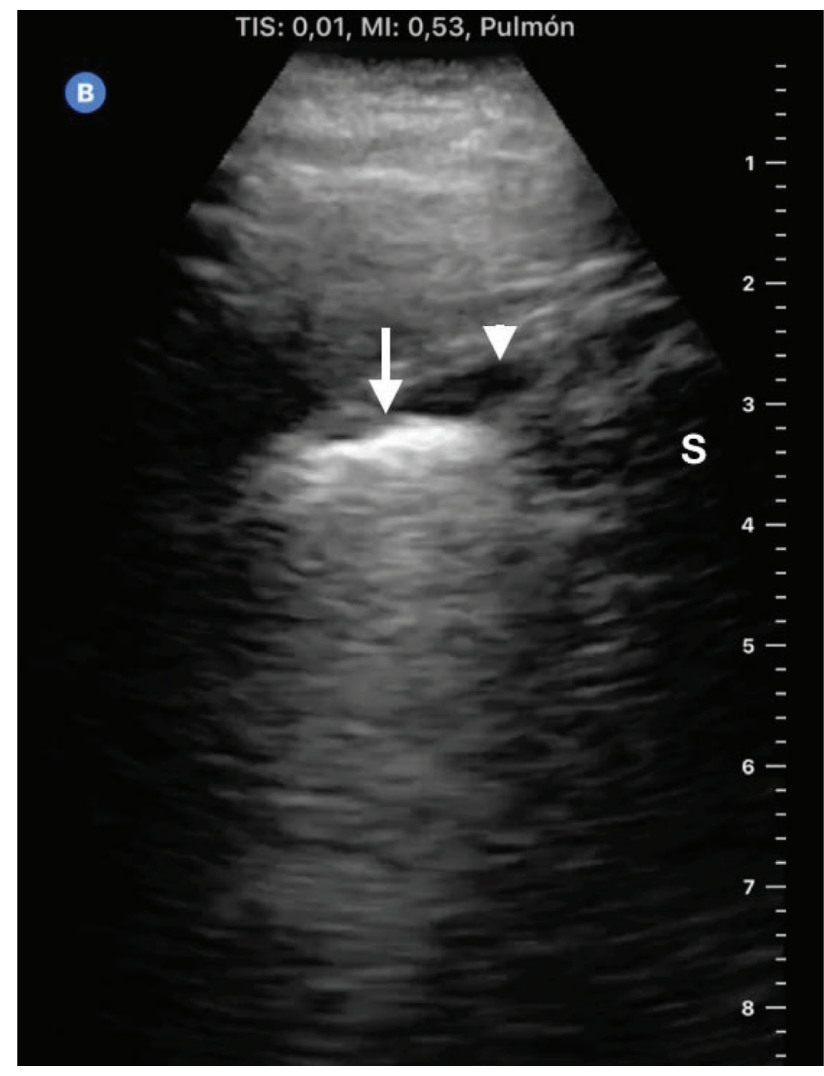

Fig 2. Day 2 - curvilinear probe. Left posterior lower lobe of the lung. Irregular pleural line (arrow). Small pleural effusion (arrowhead). S: spleen.

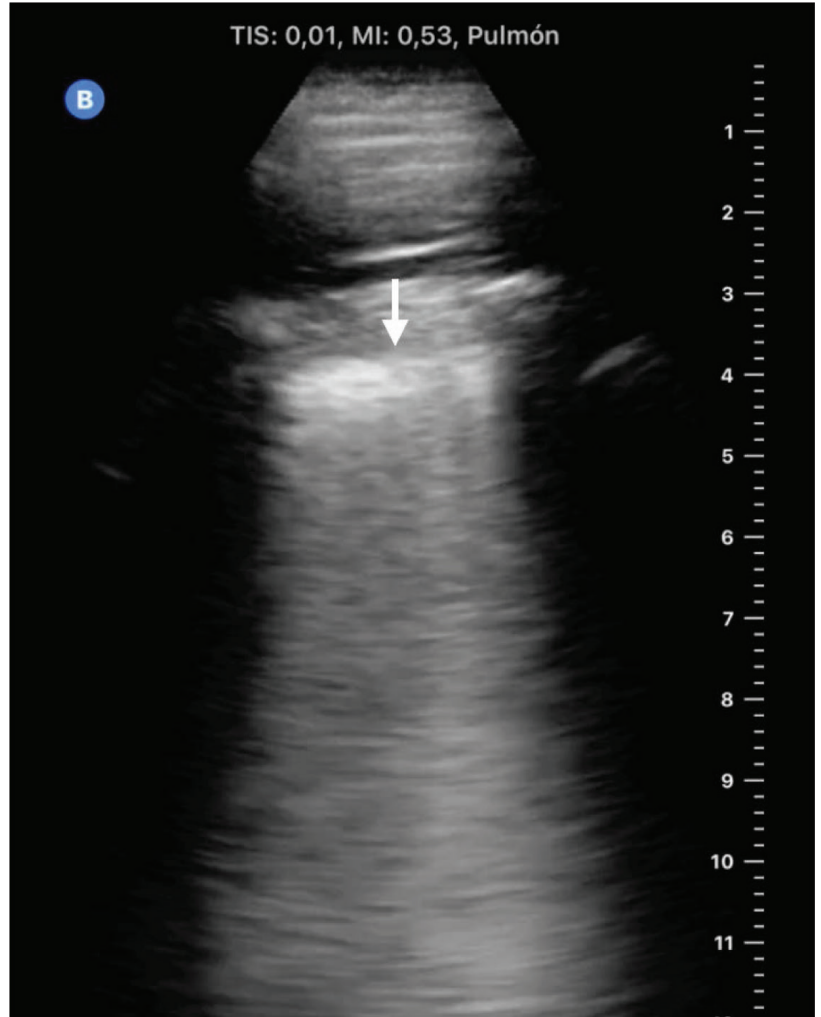

Fig 3. Day 4 - curvilinear probe. Left posterior lower lobe of the lung. Subpleural consolidation (arrow) and small pleural effusion (arrowhead). S: spleen.

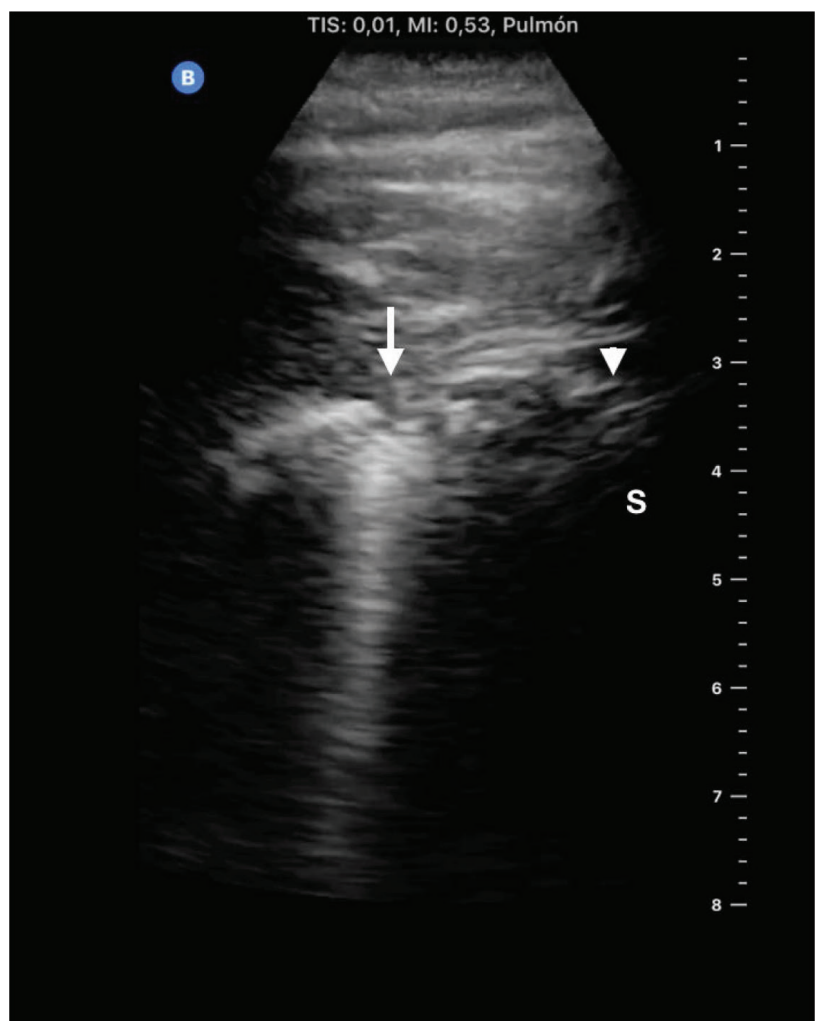

Fig 4. Day 10 - curvilinear probe. Left posterior lower lobe of the lung. B-lines (arrow). 


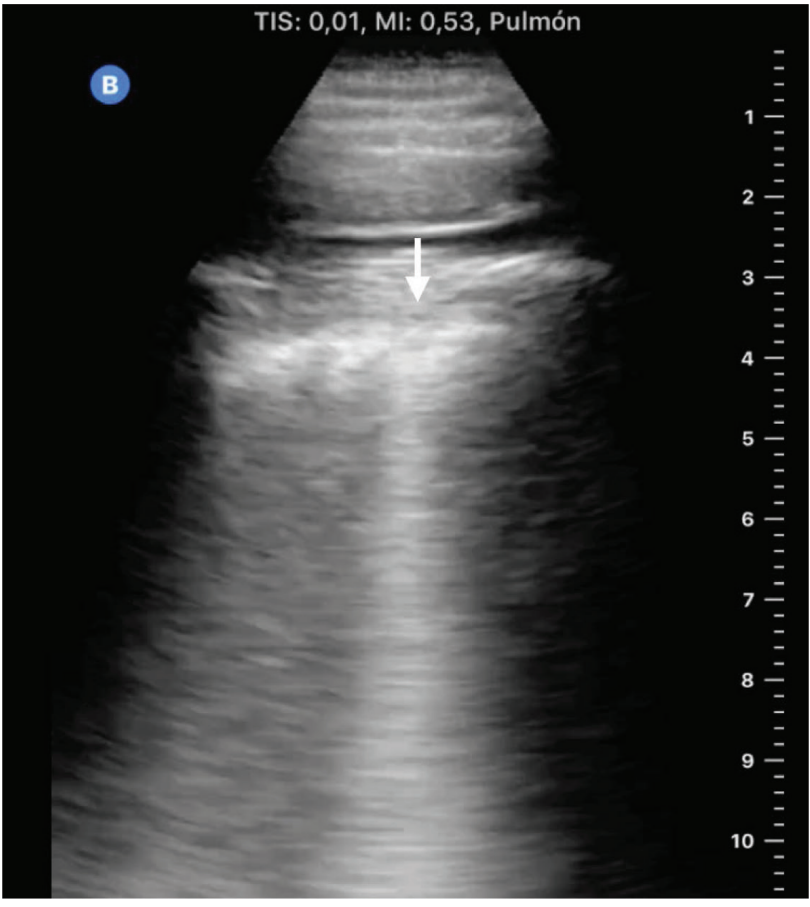

Fig 5. Day 14 - curvilinear probe. Left posterior lower lobe of the lung. Irregular pleural line and B-lines (arrow).
LUS-based close monitoring at home. Supportive therapy was started with ibuprofen $600 \mathrm{mg}$ and paracetamol $1 \mathrm{~g}$ (both three times a day). After confirming worsening of symptoms and LUS findings, hydroxychloroquine $200 \mathrm{mg}$ twice a day and azithromycin $250 \mathrm{mg}$ once daily was started for 7 days (off-label use). ${ }^{6}$

The patient's condition improved after 2 weeks from symptom onset along with improved ultrasound findings. The subpleural consolidations resolved and solitary B-lines and a small plaque of pleural thickening remained (Fig 5), but were replaced by A-lines after an additional 3 weeks (Fig 6).

\section{Discussion}

There is growing evidence regarding the imaging findings of COVID-19. The most common form of radiographic presentation is the presence of a local or bilateral patchy shadowing infiltrate on chest X-ray, although $\mathrm{X}$-ray has a poor sensitivity for detecting abnormalities (absent in more than $40 \%$ of the cases). ${ }^{4}$ Computed tomography (CT), with higher sensitivity, show ground glass opacities, ${ }^{7}$ which is the reason why CT had been proposed as the main imaging test and incorporated into different therapeutic and triage strategies since the start of the outbreak. ${ }^{8}$

While CT of the chest are more sensitive for thoracic disease there are some notable drawbacks. For mild illness, radiation exposure and overuse of healthcare resources, or lack of availability to perform a CT, seems to overshadow the need. In

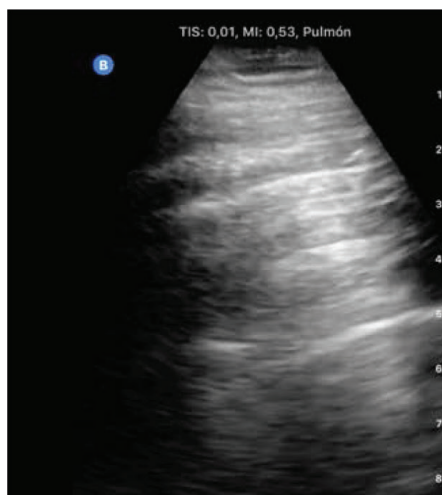

Day 1

A-lines

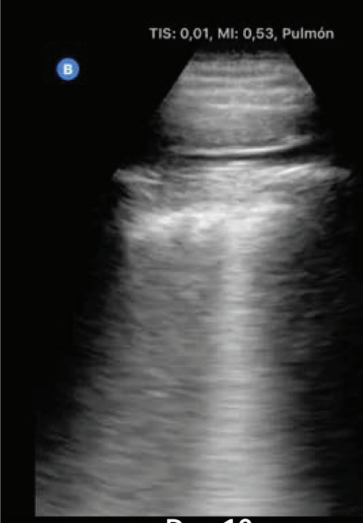

Day 10

Diffuse B-lines

10

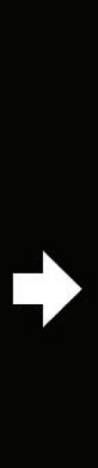

Day 2

Pleural effusion
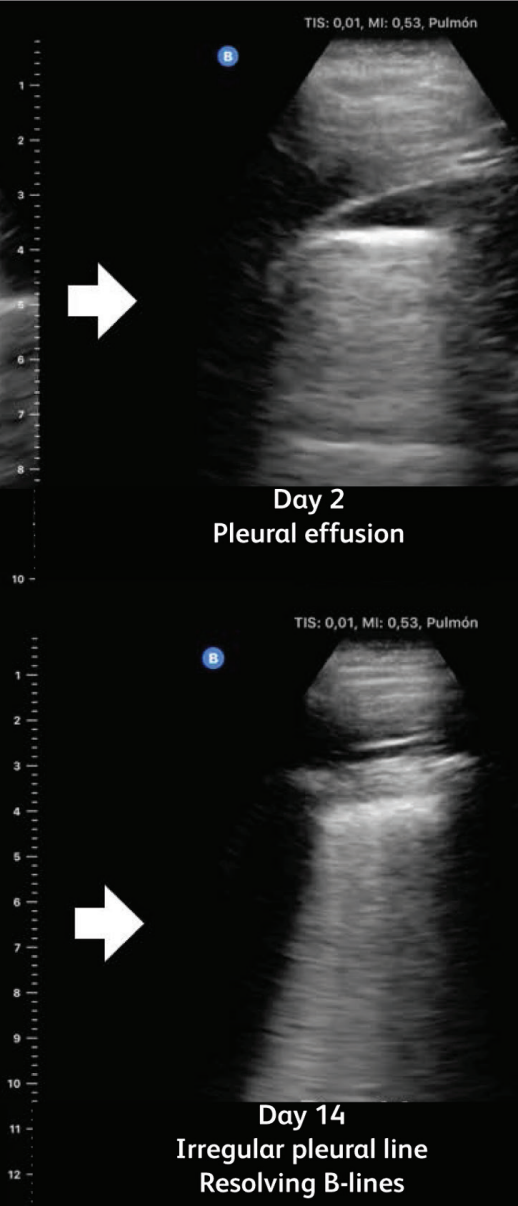

2

ion 
the critically ill, the nonspecific nature of the radiographic disease, coupled with the transport of unstable patients and exposure of infected patients, may also outweigh the clinical benefit.

Ultrasound machines are widely available and therefore LUS can be performed in a few minutes, in mild or even unstable patients, at home or in hospital settings..$^{9,10}$ Moreover, this type of imaging modality is dynamic and can be easily repeated, providing real-time decision support in the management of the patient. Although there is an ongoing debate about how it should be applied, there is a general consensus about its usefulness..$^{11,12}$ COVID-19 is likely to have the same ultrasonography findings as other viral lower respiratory tract infections. There were several studies carried out in children and adults during the H1N1 pandemic and the main sonographic finding was the presence of B-lines and subpleural consolidations..$^{13,14}$ Other studies have shown that LUS is superior to clinical evaluation and chest radiography for the diagnosis of lung involvement. ${ }^{15}$ The findings in this report raise the question of whether the severity of LUS findings can be used to differentiate minor from severe disease in this pandemic, or if home ultrasonography could be a novel care path worth exploring for borderline patients as a means of extending hospital capacity.

The presence of subpleural consolidations and B-lines are highly specific for interstitial syndrome, parenchymal or pleural disease. In this outbreak scenario they suggest the presence of COVID-19 pneumonia, and finding them could predict symptom worsening. This work differs from other available reports ${ }^{16}$ as it describes sequential evolution in more detail. However, the main limitation is that these findings overlap with those from other pneumonia aetiologies, and caution is advised in the interpretation. Other severe complications such as ARDS, could also easily be suspected with the finding of nonhomogeneous distribution of B-lines and 'spared areas' of normal lung parenchyma. ${ }^{10}$

In conclusion, ultrasound is more available than $\mathrm{CT}$ or $\mathrm{X}$-ray, and allows us to better understand the pathophysiology of the disease. With this case report we emphasise the utility and the opportunity that LUS presents in some clinical scenarios, like this COVID-19 pandemic, and how it may serve as a monitoring and therapy guide.

\section{References}

1 World Health Organization. Pneumonia of unknown cause - China disease outbreak news, 2020. www.who.int/csr/don/05-january2020-pneumonia-of-unkown-cause-china/en/ [Accessed 29 April 2020].

2 World Health Organization. Rolling updates on coronavirus (COVID19). WHO characterizes COVID-19 as a pandemic, 2019-20. www.who.int/emergencies/diseases/novel-coronavirus-2019/ events-as-they-happen [Accessed 29 April 2020].

3 Fauci AS, Lane HC, Redfield RR. Covid-19 - navigating the uncharted. N Engl J Med 2020;382:1268-69.

4 Guan W, Ni Z, Hu Y et al. Clinical characteristics of coronavirus disease 2019 in China. N Engl J Med 2020;NEJMoa2002032.

5 Volpicelli G, Mussa A, Garofalo G et al. Ultrasonido pulmonar de cabecera en la evaluación del síndrome alveolar-intersticial. Am J Emerg Med 2006;24:689-96.

6 Gautret P, Lagier J-C, Parola P et al. Hydroxychloroquine and azithromycin as a treatment of COVID-19: results of an open-label non-randomized clinical trial. Int ] Antimicrob Agents 2020;105949.

7 Shi $\mathrm{H}, \mathrm{Han} \mathrm{X}$, Jiang $\mathrm{N}$ et al. Radiological findings from 81 patients with COVID-19 pneumonia in Wuhan, China: a descriptive study. Lancet Infect Dis 2020;20:425-34.

8 Zhang J, Zhou L, Yang Y et al. Therapeutic and triage strategies for 2019 novel coronavirus disease in fever clinics. Lancet Respir Med 20208:e11-2.

9 Ultrasound Guidelines: Emergency, point-of-care and clinical ultrasound guidelines in medicine. Ann Emerg Med 2017;69:e27-54.

10 Volpicelli G, Elbarbary M, Blaivas M et al. International evidence-based recommendations for point-of-care lung ultrasound. Intensive Care Med 2012;38:577-91.

11 Walter JM, Satterwhite L, Lyn-Kew KE. POINT: Should the use of diagnostic point-of-care ultrasound in patient care - require hospital privileging/credentialing? Yes. Chest 2020;157:496-8.

12 Kruser JM, Schmidt GA, Kory PD. COUNTERPOINT: Should the use of diagnostic point-of-care ultrasound in patient care require hospital privileging/credentialing? No. Chest 2020;157:498-500.

13 Tsung JW, Kessler DO, Shah VP. Prospective application of clinician-performed lung ultrasonography during the 2009 H1N1 influenza A pandemic: distinguishing viral from bacterial pneumonia. Crit Ultrasound ] 2012;4:16.

14 Testa A, Soldati G, Copetti R et al. Early recognition of the 2009 pandemic influenza A (H1N1) pneumonia by chest ultrasound. Crit Care Lond Engl 2012;16:R30.

15 Tierney DM, Huelster JS, Overgaard JD et al. Comparative performance of pulmonary ultrasound, chest radiograph and CT among patients with acute respiratory failure. Crit Care Med 2020;48:151-7.

16 Huang Y, Wang S, Liu Y et al. A preliminary study on the ultrasonic manifestations of peripulmonary lesions of non-critical novel coronavirus pneumonia (COVID-19). SSRN, 2020. Available from https://ssrn.com/abstract=3544750.

Address for correspondence: Dr Yale Tung Chen, Servicio de Urgencias. Hospital Universitario La Paz. Paseo de la Castellana, 261, 28046 Madrid, Spain.

Email:yale.tung@salud.madrid.org 